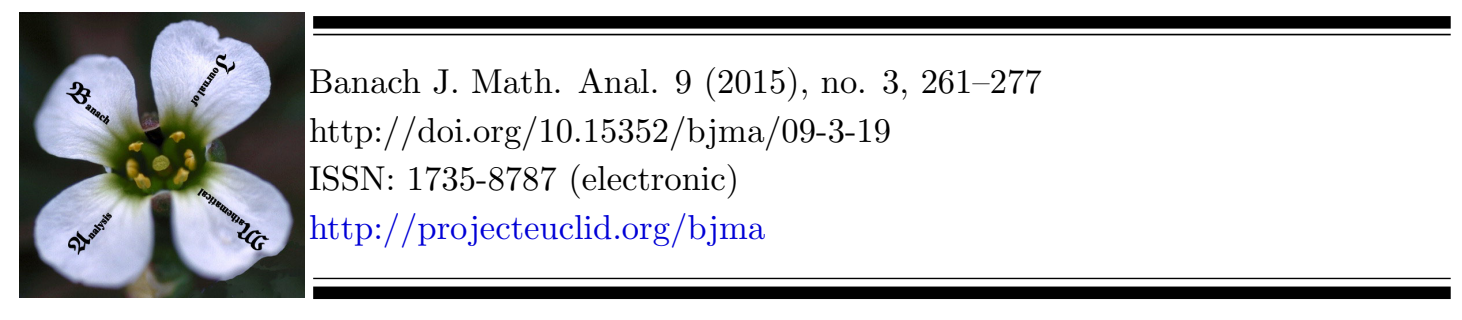

\title{
HILBERT SPACE REPRESENTATIONS OF THE QUANTUM *-ALGEBRA $\mathcal{U}_{q}\left(s u_{1,1}\right)$ DAVID DUBRAY
}

Communicated by M. Joiţa

\begin{abstract}
The Hilbert space representation theory of the $q$-deformed quantum $*$-algebra $\mathcal{U}_{q}\left(s u_{1,1}\right)$ is studied using the inducing procedure. As a result we obtain four series of irreducible induced $*$-representations on Hilbert spaces. Furthermore, we show that there is a one-to-one correspondence between the induced series and the series of irreducible well-behaved $*$-representations of $\mathcal{U}_{q}\left(s u_{1,1}\right)$ computed by Burban and Klymik.
\end{abstract}

\section{INTRODUCTION}

The representation theory of groups was founded by G. Frobenius in 1898. In his work [5], he constructed induced representations of finite matrix groups from subgroups. Subsequently, his work was extended and systematically developed by G.W. Mackey in [7].

In [9], Y. Savchuk and K. Schmüdgen developed a theory of unbounded induced Hilbert space representations. Important parts of this theory are a definition of well-behaved representations, several versions of the imprimitivity theorem, a generalization of Mackey's analysis and an inducing procedure for group graded $*$-algebras and one-dimensional $*$-representations. Examples of $*$-representations of $*$-algebras which are induced from a commutative $*$-subalgebra are given in [9] and in the recent paper [3] by Y. Savchuk and P. Dowerk.

The aim of the present paper is a detailed analysis of the inducing procedure for a much more involved example: the quantum $*$-algebra $\mathcal{U}_{q}\left(s u_{1,1}\right)$. This $*$-algebra

Date: Received: Oct. 13, 2014; Accepted: Nov. 18, 2014.

2010 Mathematics Subject Classification. Primary 47L55; Secondary 16G99, 16W50, 47L60.

Key words and phrases. Induced representations, group graded algebras, well-behaved representations, q-deformed enveloping algebra. 
has four series of unbounded irreducible series of $*$-representations. In 1993, Burban and Klymik [2] computed all irreducible $*$-representations of $\mathcal{U}_{q}\left(s u_{1,1}\right)$ which fulfill certain technical regularity conditions. We elaborate all $*$-representations of the $*$-algebra $\mathcal{U}_{q}\left(s u_{1,1}\right)$ which are induced by characters of the zero $*$-subalgebra with the grading defined by

$$
E \in \mathcal{A}_{1} \text { and } K, K^{-1} \in \mathcal{A}_{0} .
$$

The equivalence classes of induced $*$-representations are parametrized by means of the positive characters. In our main theorem, we obtain a one-to-one correspondence between subfamilies of equivalence classes of induced $*$-representations and the series of $*$-representations derived in [2]. This result gives new insight into the inducing procedure and another perspective on the representation theory of the quantum $*$-algebra $\mathcal{U}_{q}\left(s u_{1,1}\right)$.

For a more general approach to induced representations we refer to [4]. Important monographs on the general theory of Hilbert space representations are [1], [8], [6] and [11]. The quantum space $U_{q}\left(s l_{2}\right)$ was introduced in [10]. The *-algebra $\mathcal{U}_{q}\left(s u_{1,1}\right)$ is a real form of this quantum algebra.

In the following preliminaries, we will introduce the inducing procedure given in [9] with some minor simplifications fitting our setting.

0.1. Preliminaries. In this work an algebra is always an associative algebra with unit over the field $\mathbb{C}$. A $*$-algebra $\mathcal{A}$ is an algebra with involution, i.e. a conjugate linear map $*: \mathcal{A} \rightarrow \mathcal{A}, a \mapsto a^{*}$ fulfilling $a^{* *}=a$ and $(a b)^{*}=b^{*} a^{*}$ for all $a, b \in \mathcal{A}$. For a better reading experience, we will use negative exponents in the following way: we denote $\left(a^{*}\right)^{n}$ by $a^{-n}$ for $n \in \mathbb{N}$. The zero exponent is defined by $a^{0}:=1_{\mathcal{A}}$. With this notation the exponentiation rule $a^{n+m}=a^{n} a^{m}, m, n \in \mathbb{Z}$, does not hold in general.

We denote the set of all linear operators from a linear vector space $X$ over $\mathbb{C}$ to itself by $L(X)$. Let $\mathcal{D}$ be a dense linear subspace of a Hilbert space $\mathcal{H}$. An algebra homomorphism $\pi: \mathcal{A} \rightarrow L(\mathcal{D})$ is called a *-representation if

$$
\langle\pi(a) \varphi, \psi\rangle_{\mathcal{H}}=\left\langle\varphi, \pi\left(a^{*}\right) \psi\right\rangle_{\mathcal{H}}
$$

for all $\varphi, \psi \in \mathcal{D}, a \in \mathcal{A}$. We denote the corresponding dense linear subspace to a $*$-representation $\pi$ by $\mathcal{D}(\pi)$ and the corresponding Hilbert space by $\mathcal{H}(\pi)$. Occasionally, we call $\mathcal{D}(\pi)$ the domain of $\pi$ and $\mathcal{H}(\pi)$ the carrier Hilbert space of $\pi$.

The graph topology of a *-representation $\pi$ is the local convex topology on the vector space $\mathcal{D}(\pi)$ induced by the family of semi-norms

$$
\{\|\cdot\|+\|\pi(a) \cdot\|\}_{a \in \mathcal{A}}
$$

The closure of a $*$-representation $\pi$ is the $*$-representation $\bar{\pi}$ defined by $\bar{\pi}(a):=$ $\overline{\pi(a)} \uparrow_{\mathcal{D}(\bar{\pi})}, a \in \mathcal{A}$, where $\mathcal{D}(\bar{\pi})$ is the closure of $\mathcal{D}(\pi)$ in the graph topology and $\overline{\pi(a)}$ is the closure of $\pi(a)$ as an operator densely defined on $\mathcal{H}(\pi)$. A *representation $\pi$ is closed if $\pi=\bar{\pi}$.

The direct sum of two $*$-representations $\pi_{1}, \pi_{2}$ is denoted by $\pi_{1} \oplus \pi_{2}$. It is defined on the direct $\operatorname{sum} \mathcal{D}\left(\pi_{1}\right) \oplus \mathcal{D}\left(\pi_{2}\right)$ and maps $a \mapsto \pi_{1}(a) \oplus \pi_{2}(a)$. A *-representation $\pi$ 
is called irreducible if for every two $*$-representations $\pi_{1}, \pi_{2}$ with $\pi=\pi_{1} \oplus \pi_{2}$ one of the domains $\mathcal{D}\left(\pi_{1}\right)$ and $\mathcal{D}\left(\pi_{2}\right)$ is zero. Two $*$-representations $\pi_{1}, \pi_{2}$ of a $*$-algebra $\mathcal{A}$ are called unitarily equivalent if there is a unitary operator $U: \mathcal{D}\left(\pi_{1}\right) \longrightarrow \mathcal{D}\left(\pi_{2}\right)$ fulfilling $U^{-1} \pi_{2}(a) U=\pi_{1}(a)$ for all $a \in \mathcal{A}$.

For an arbitrary set $I$, we denote the direct sum of a family of linear spaces $\left\{X_{i}\right\}_{i \in I}$ by $\oplus_{i \in I} X_{i}$.

Definition 0.1. Let $(G,+)$ be a (discrete) group. A $G$-graded *-algebra $\mathcal{A}$ is a *-algebra with linear subspaces $\mathcal{A}_{g} \subset \mathcal{A}, g \in G$, such that

$$
\begin{array}{ll}
\mathcal{A}=\oplus_{g \in G} \mathcal{A}_{g}, & \\
\mathcal{A}_{g_{1}} \mathcal{A}_{g_{2}} \subset \mathcal{A}_{g_{1}+g_{2}} & \forall g_{1}, g_{2} \in G, \\
\mathcal{A}_{g}^{*} \subset \mathcal{A}_{-g} & \forall g \in G .
\end{array}
$$

The linear subspace $\mathcal{A}_{0}$ of the neutral element of the group will be called the zero $*$-subalgebra of $\mathcal{A}$, which, indeed, is a $*$-subalgebra by properties $(0.2)$ and (0.3). We call the elements of a linear subspace $\mathcal{A}_{g}, g \in G$, homogeneous elements.

We call the quadratic module $\sum \mathcal{A}^{2}$ the finite sums of squares. It is defined by

$$
\sum \mathcal{A}^{2}:=\left\{\sum_{i=1}^{n} a_{i}^{*} a_{i} \mid a_{1}, \ldots, a_{n} \in \mathcal{A}, n \in \mathbb{N}\right\} .
$$

The subset $\sum \mathcal{A}^{2} \cap \mathcal{A}_{0}$ of the finite sums of squares of $\mathcal{A}$ is called the positive cone of $\mathcal{A}_{0}$. Its structure is described in the following lemma.

Lemma 0.2. An element $a \in \mathcal{A}_{0}$ belongs to the positive cone $\sum \mathcal{A}^{2} \cap \mathcal{A}_{0}$ if and only if it can be presented as a finite sum of squares $\sum_{x \in X} x^{*} x$ such that $X \subset \bigcup_{g \in G} \mathcal{A}_{g}$.

Let us assume that the zero $*$-subalgebra $\mathcal{A}_{0}$ of $\mathcal{A}$ is commutative and denote it by $\mathcal{B}$. We call a non-trivial $*$-representation of $\mathcal{B}$ onto $\mathbb{C}$ a character. A character $\chi$ is positive if it is non-negative on the positive cone of $\mathcal{B}$, i.e. if $\chi\left(\sum \mathcal{A}^{2} \cap \mathcal{B}\right) \subseteq$ $\mathbb{R}_{0}^{+}$.

If for every $g \in G$ there is an element $c_{g} \in \mathcal{A}_{g}$ such that $\mathcal{A}_{g}=c_{g} \mathcal{B}$, then $\chi$ is positive if and only if

$$
\chi\left(c_{g}^{*} c_{g}\right) \geq 0 \text { for all } g \in G .
$$

The set of characters will be denoted by $\widehat{\mathcal{B}}$ and the set of positive characters by $\widehat{\mathcal{B}}^{+}$. From [9] follows that for every positive character there is an induced *-representation $\operatorname{Ind}_{\chi}$ of $\mathcal{A}$.

Definition 0.3. Let $\chi \in \widehat{\mathcal{B}}^{+}$and $g \in G$. If there is an element $a_{g} \in \mathcal{A}_{g}$ such that $\chi\left(a_{g}^{*} a_{g}\right) \neq 0$, we define the map $\chi^{g}: \mathcal{B} \rightarrow \mathbb{C}$ by

$$
\chi^{g}(b):=\frac{\chi\left(a_{g}^{*} b a_{g}\right)}{\chi\left(a_{g}^{*} a_{g}\right)} \text { for all } b \in \mathcal{B} .
$$

Otherwise, we say that $\chi^{g}$ is not defined. For an element $g \in G$ we denote by $\mathcal{D}_{g}$ the set of all characters $\chi \in \widehat{\mathcal{B}}^{+}$such that $\chi^{g}$ is defined. 
Lemma 0.4. Let $\chi \in \widehat{\mathcal{B}}^{+}, g \in G$ and suppose $\chi^{g}$ is defined. Then $\chi^{g}$ is a positive character.

Let us introduce three important definitions related to the partial group action. For $\chi \in \widehat{\mathcal{B}}^{+}$the subset $G_{\chi} \subset G$ is defined by

$$
G_{\chi}:=\left\{g \in G \mid \chi^{g} \text { is defined }\right\},
$$

the orbit of $\chi$ is defined by

$$
\operatorname{Orb}_{\chi}:=\left\{\chi^{g} \mid \chi^{g} \text { is defined }\right\}
$$

and the stabilizer of $\chi$ is defined by

$$
\mathrm{St}_{\chi}:=\left\{g \in G \mid \chi^{g} \text { is defined and equal to } \chi\right\} \text {. }
$$

Obviously, we have $0 \in \mathrm{St}_{\chi} \cap G_{\chi}$ and $\chi \in \mathrm{Orb}_{\chi}$.

Proposition 0.5. Let $\chi \in \widehat{\mathcal{B}}^{+}$. If $\psi \in \widehat{\mathcal{B}}^{+}$, then $O r b_{\psi} \cap O r b_{\chi} \neq \emptyset$ if and only if $\mathrm{Orb}_{\psi}=\mathrm{Orb}_{\chi}$.

The next proposition gives explicit formulas for induced $*$-representations by characters. Recall that the representation space $\mathcal{H}(\pi)$ of an induced $*$-representation $\pi=\operatorname{Ind}_{\chi}, \chi \in \widehat{\mathcal{B}}^{+}$, is spanned by the vectors $[a \otimes 1], a \in \mathcal{A}$.

Proposition 0.6. Let $\chi \in \widehat{\mathcal{B}}^{+}$and set $\pi:=$ Ind $_{\chi}$. For all $g \in G_{\chi}$ fix elements $a_{g} \in \mathcal{A}_{g}$ such that $\chi\left(a_{g}^{*} a_{g}\right) \neq 0$.

[(i)]The vectors

$$
e_{g}=\frac{\left[a_{g} \otimes 1\right]}{\sqrt{\chi\left(a_{g}^{*} a_{g}\right)}}, g \in G_{\chi}
$$

form an orthonormal basis of the carrier Hilbert space $\mathcal{H}(\pi)$. For $c_{h} \in$ $\mathcal{A}_{h}, h \in G$, we have

$$
\pi\left(c_{h}\right) e_{g}=\frac{\chi\left(a_{h+g}^{*} c_{h} a_{g}\right)}{\sqrt{\chi\left(a_{h+g}^{*} a_{h+g}\right) \chi\left(a_{g}^{*} a_{g}\right)}} e_{h+g} \quad \text { if } h+g \in G_{\chi}
$$

and $\pi\left(c_{h}\right) e_{g}=0$ if $h+g \notin G_{\chi}$. In particular, if $b \in \mathcal{B}$, then we have

$$
\pi(b) e_{g}=\frac{\chi\left(a_{g}^{*} b a_{g}\right)}{\chi\left(a_{g}^{*} a_{g}\right)} e_{g}=\chi^{g}(b) e_{g} .
$$

For the next two propositions let $\mathcal{B}$ be countably generated and let $G$ be countable.

Proposition 0.7. An induced $*$-representation Ind $_{\chi}, \chi \in \widehat{\mathcal{B}}^{+}$, is irreducible if and only if the stabilizer $S t_{\chi}$ is trivial.

Proposition 0.8. Two induced *-representations $\operatorname{Ind}_{\chi}, \operatorname{Ind}_{\psi}, \psi, \chi \in \widehat{\mathcal{B}}^{+}$, are unitarily equivalent if and only if $\mathrm{Orb}_{\chi}=\mathrm{Orb}_{\psi}$. 


\section{The Quantum *-AlgeBra $\mathcal{U}_{q}\left(\mathrm{SU}_{1,1}\right)$}

We shall induce $*$-representations of the quantum $*$-algebra $\mathcal{U}_{q}\left(\mathrm{su}_{1,1}\right)$ using a $\mathbb{Z}$-grading. Next, we compare the induced $*$-representations with the different series of $*$-representations from I.M. Burban and A.U. Klimyk in [2]. These *-representations will be referred to as the classical or well-behaved $*$-representations or series.

Using a parametrization based on the computation of the positive characters, we will be able to show a one-to-one correspondence of the induced equivalence classes of $*$-representations and the classical series. Moreover, we will be able to assign subsets of the induced equivalence classes to the various corresponding classical series.

1.1. Definition and $\mathbb{Z}$-Grading. In this section suppose $q \in(0,1)$ is fixed. The *-algebra $\mathcal{U}_{q}\left(\mathrm{su}_{1,1}\right)$ has the generators $E, F, K, K^{-1}$ with the defining relations

$$
\begin{aligned}
K K^{-1} & =K^{-1} K=1, \\
K E K^{-1} & =q E, \quad K F K^{-1}=q^{-1} F, \\
{[E, F] } & =\frac{K^{2}-K^{-2}}{q-q^{-1}}
\end{aligned}
$$

and an involution defined by

$$
E^{*}=-F, F^{*}=-E, K^{*}=K, K^{-1^{*}}=K^{-1} .
$$

Let us define a $\mathbb{Z}$-grading on $\mathcal{A}:=\mathcal{U}_{q}\left(\mathrm{su}_{1,1}\right)$ by

$$
E \in \mathcal{A}_{1} \text { and } K, K^{-1} \in \mathcal{A}_{0} .
$$

Looking at the defining relations and the involution of $\mathcal{A}$, we see that this grading is well-defined. Additionally, we see:

[(i)] The zero $*$-subalgebra $\mathcal{B}:=\mathcal{A}_{0}=\mathbb{C}\left[E F, K, K^{-1}\right]$ is commutative.

The linear subspaces of the $\mathbb{Z}$-grading are $\mathcal{A}_{z}=E^{z} \mathcal{B}, z \in \mathbb{Z}$.

From the theory of quantum $*$-algebras it is well-known that the quantum Casimir element of $\mathcal{U}_{q}\left(\mathrm{su}_{1,1}\right)$ is

$$
C_{q}=E F+\frac{q^{-1} K^{2}+q K^{-2}}{\left(q-q^{-1}\right)^{2}} .
$$

Since the quantum Casimir element is part of the centralizer of $\mathcal{U}_{q}\left(\mathrm{su}_{1,1}\right)$ and $E F \in \mathbb{C}\left[C_{q}, K, K^{-1}\right]$, the zero $*$-subalgebra is $\mathcal{B}=\mathbb{C}\left[C_{q}, K, K^{-1}\right]$.

1.2. Inducing Procedure - First Part. Due to the generators of $\mathcal{B}$ each character $\chi$ is determined by two parameters $(s, t) \in \mathbb{C} \times \mathbb{C}_{\neq 0}$ if $\chi\left(C_{q}\right)=s$ and $\chi(K)=t$. Therefore, we parametrize the characters by such tuples, and write $\chi_{s, t}$ for an element of $\widehat{\mathcal{B}}$. If $\chi_{s, t}$ is in $\widehat{\mathcal{B}}^{+}$, we denote the induced $*$-representation of $\chi_{s, t}$ by $\pi_{s, t}$.

Let $\chi$ be a positive character. To simplify notation, we denote an equivalence class $[A \otimes \lambda] \in \mathcal{A} \otimes_{\mathcal{B}} \mathbb{C} / \mathcal{K}_{\chi}$ by its representative $A \otimes \lambda$. The one or zero dimensional linear subspace of $\mathcal{A} \otimes_{\mathcal{B}} \mathbb{C} / \mathcal{K}_{\chi}$ spanned by $\left\{a_{z} \otimes 1 \mid a_{z} \in \mathcal{A}_{z}\right\}$ will be denoted by $\mathcal{A}_{z} \otimes \mathbb{C}$ for $z \in \mathbb{Z}$. 
For the generators $K$ and $K^{-1}$, since $K^{*}=K$ and $K^{-1^{*}}=K^{-1}$, we will use the slightly inconsistent but intuitive notation $K^{-z}=\left(K^{-1}\right)^{z}$ for $z \in \mathbb{Z}$.

As it is usual in this context, we use the notation of the $q$-Calculus $[z]=[z]_{q}=$ $\frac{q^{z}-q^{-z}}{q-q^{-1}}$ for $z \in \mathbb{Z}$ and write adapted to our special case $\left[K^{2} ; z\right]=\left[K^{2} ; z\right]_{q}=$ $\frac{q^{z} K^{2}-q^{-z} K^{-2}}{q-q^{-1}}, z \in \mathbb{Z}$.

The following lemma is a well-known fact from the analysis of the quantum *-algebra $\mathcal{U}_{q}\left(\mathrm{su}_{2}\right)$ :

Lemma 1.1. For all $n \in \mathbb{N}$, we have the equalities

$$
\left[E, F^{n}\right]=F^{n-1}[n]\left[K^{2} ; 1-n\right] \quad \text { and } \quad\left[E^{n}, F\right]=E^{n-1}[n]\left[K^{2} ; n-1\right]
$$

in $\mathcal{U}_{q}\left(s u_{2}\right)$ and $\mathcal{U}_{q}\left(s u_{1,1}\right)$.

Lemma 1.2. Let $z \in \mathbb{Z}$. The following equations hold:

$$
\begin{aligned}
E^{* z} E^{z} & =\prod_{k=1}^{z}\left([k]\left[K^{2} ; k-1\right]-E F\right) \text { for } z>0, \\
\text { and } \quad E^{* z} E^{z} & =\prod_{k=1}^{|z|}\left([1-k]\left[K^{2} ;-k\right]-E F\right) \text { for } z<0 .
\end{aligned}
$$

(2) Proof. We use Lemma 1.1 and show exemplary the case $z>0$.

For $z>0$ we have

$$
\begin{aligned}
E^{* z} E^{z} & =(-F)^{z} E^{z}=(-1)^{z} F^{z-1}\left(E^{z} F-E^{z-1}[z]\left[K^{2} ; z-1\right]\right) \\
& =(-1)^{z} \prod_{k=1}^{z}\left(E F-[k]\left[K^{2} ; k-1\right]\right)=\prod_{k=1}^{z}\left([k]\left[K^{2} ; k-1\right]-E F\right) .
\end{aligned}
$$

Lemma 1.3. A character $\chi_{s, t}$ is in $\widehat{\mathcal{B}}^{+}$if and only if

$$
\chi_{s, t}\left(E^{* n} E^{n}\right)=\prod_{k=1}^{n}\left(\frac{q^{2 k-1} t^{2}+q^{-2 k+1} t^{-2}}{\left(q-q^{-1}\right)^{2}}-s\right) \geq 0
$$

and

$$
\chi_{s, t}\left(E^{n} E^{* n}\right)=\prod_{k=1}^{n}\left(\frac{q^{2 k-1} t^{-2}+q^{-2 k+1} t^{2}}{\left(q-q^{-1}\right)^{2}}-s\right) \geq 0
$$

for all $n \in \mathbb{N}$.

Proof. Since $\mathcal{A}_{z}=E^{z} \mathcal{B}$ for all $z \in \mathbb{Z}$, we can use condition (0.4). Hence, a character is positive if it is non-negative on the terms $E^{z^{*}} E^{z}, z \in \mathbb{Z}$.

The factors of (1.1) are

$$
\begin{aligned}
{[k]\left[K^{2} ; k-1\right]-E F } & =\frac{q^{2 k-1} K^{2}-q^{-1} K^{2}-q K^{-2}+q^{-2 k+1} K^{-2}}{\left(q-q^{-1}\right)^{2}}-E F \\
& =\frac{q^{2 k-1} K^{2}+q^{-2 k+1} K^{-2}}{\left(q-q^{-1}\right)^{2}}-C_{q}
\end{aligned}
$$


and the factors of (1.2) are

$$
\begin{aligned}
{[1-k]\left[K^{2} ;-k\right]-E F } & =\frac{q^{-2 k+1} K^{2}-q^{-1} K^{2}-q K^{-2}+q^{2 k-1} K^{-2}}{\left(q-q^{-1}\right)^{2}}-E F \\
& =\frac{q^{2 k-1} K^{-2}+q^{-2 k+1} K^{2}}{\left(q-q^{-1}\right)^{2}}-C_{q}
\end{aligned}
$$

Applying $\chi_{s, t}$ on the right-hand side of (1.1) and (1.2) shows the assertion.

Lemma 1.4. If $\chi_{s, t} \in \widehat{\mathcal{B}}^{+}$, then $(s, t) \in \mathbb{R} \times \mathbb{R}_{\neq 0}$.

Proof. Let $\chi_{s, t} \in \widehat{\mathcal{B}}^{+}$. We have $\chi_{s, t}\left(E E^{*}\right)=-\chi_{s, t}(E F) \geq 0$, which implies that $\chi_{s, t}(E F)$ is real. Since $\chi_{s, t}\left(K^{2}\right)=t^{2} \geq 0, t^{2}$ and $t$ are real. Further, since $C_{q}=E F+\frac{q^{-1} K^{2}+q K^{-2}}{\left(q-q^{-1}\right)^{2}}, s=\chi_{s, t}\left(C_{q}\right)$ is real.

In the following, if a character $\chi_{s, t}$ or a $*$-representation $\pi_{s, t}$ is introduced, we assume that $(s, t) \in \mathbb{R} \times \mathbb{R}_{\neq 0}$ if the pair $(s, t)$ is not defined explictly.

For every induced $*$-representation $\pi_{s, t}$, we define

$$
\begin{gathered}
e_{k}:=\frac{E^{k} \otimes 1}{\left\|E^{k} \otimes 1\right\|} \\
\text { and } \quad M_{s, t}:=G_{\chi_{s, t}}=\left\{k \in \mathbb{Z} \mid E^{k} \otimes 1 \neq 0 \text { in } \mathcal{H}\left(\pi_{s, t}\right)\right\} .
\end{gathered}
$$

Then, by Proposition 0.6, the family $\left\{e_{k}\right\}_{k \in M_{s, t}}$ is an orthonormal system of the carrier Hilbert space $\mathcal{H}\left(\pi_{s, t}\right)$.

The action of an induced $*$-representation $\pi_{s, t}$ and the partial action of $\mathbb{Z}$ on the positive characters are described in the next two propositions.

Proposition 1.5. The induced $*$-representation $\pi_{s, t}$ has the following action on the basis vectors $e_{k}$ for all $k \in M_{s, t}$ :

$$
\begin{aligned}
& \pi_{s, t}(E) e_{k}=\left(\frac{q^{2 k+1} t^{2}+q^{-2 k-1} t^{-2}}{\left(q-q^{-1}\right)^{2}}-s\right)^{\frac{1}{2}} e_{k+1}, \\
& \pi_{s, t}(K) e_{k}=q^{k} t e_{k}
\end{aligned}
$$

where $e_{k}=0$ if $k \notin M_{s, t}$.

Proof. Let $k \in M_{s, t}$. We use Proposition 0.6 (ii).

The operator $\pi_{s, t}(K)$ is acting on $e_{k}$ by

$$
\begin{aligned}
\pi_{s, t}(K) e_{k} & =\chi_{s, t}^{k}(K) e_{k}=\frac{\chi_{s, t}\left(E^{k^{*}} K E^{k}\right)}{\chi_{s, t}\left(E^{k^{*}} E^{k}\right)} e_{k} \\
& =q^{k} \chi_{s, t}(K) e_{k}=q^{k} t e_{k} .
\end{aligned}
$$


Suppose $k+1 \in M_{s, t}$, for $k>0$ we get

$$
\begin{aligned}
\pi_{s, t}(E) e_{k} & =\frac{E^{k+1} \otimes 1}{\left\|E^{k} \otimes 1\right\|}=\frac{\left(\prod_{j=1}^{k+1}\left(\frac{q^{2 k-1} t^{2}+q^{-2 k+1} t^{-2}}{\left(q-q^{-1}\right)^{2}}-s\right)\right)^{\frac{1}{2}}}{\left(\prod_{j=1}^{k}\left(\frac{q^{2 k-1} t^{2}+q^{-2 k+1} t^{-2}}{\left(q-q^{-1}\right)^{2}}-s\right)\right)^{\frac{1}{2}}} \frac{E^{k+1} \otimes E^{k+1} \otimes 1 \|}{\|)^{\frac{1}{2}} e_{k+1},} \\
& =\left(\frac{q^{2 k+1} t^{2}+q^{-2 k-1} t^{-2}}{\left(q-q^{-1}\right)^{2}}-s\right)^{2}
\end{aligned}
$$

and for $k<0$ we get

$$
\begin{aligned}
\pi_{s, t}(E) e_{k} & =\pi_{s, t}(E) \frac{E^{k} \otimes 1}{\left\|E^{k} \otimes 1\right\|} \\
& =\frac{\chi_{s, t}\left(E^{k+1^{*}} E E^{k}\right)}{\sqrt{\chi_{s, t}\left(E^{k+1^{*}} E^{k+1}\right) \chi_{s, t}\left(E^{k^{*}} E^{k}\right)}} \frac{E^{k+1} \otimes 1}{\left\|E^{k+1} \otimes 1\right\|} \\
& =\left(\frac{\prod_{l=1}^{-k}\left(\frac{q^{2 l-1} t^{-2}+q^{-2 l+1} t^{2}}{\left(q-q^{-1}\right)^{2}}-s\right)}{\prod_{l=1}^{-k-1}\left(\frac{q^{2 l-1} t^{-2}+q^{-2 l+1} t^{2}}{\left(q-q^{-1}\right)^{2}}-s\right)}\right)^{\frac{1}{2}} e_{k+1} \\
& =\left(\frac{q^{-2 k-1} t^{-2}+q^{2 k+1} t^{2}}{\left(q-q^{-1}\right)^{2}}-s\right)^{\frac{1}{2}} e_{k+1} .
\end{aligned}
$$

If $k+1 \notin M_{s, t}$, the action stated in the assertion is obviously true.

Proposition 1.6. Let $\chi_{s, t}$ be a positive character. If $\chi_{s, t} \in \mathcal{D}_{k}$, the partial action for $k \in \mathbb{Z}$ is

$$
\begin{aligned}
& \chi_{s, t}^{k}\left(C_{q}\right)=\chi_{s, t}\left(C_{q}\right), \\
& \chi_{s, t}^{k}(K)=q^{k} \chi_{s, t}(K) .
\end{aligned}
$$

The stabilizer $S t_{\chi_{s, t}}$ of each positive character is trivial. Therefore, every induced *-representation $\pi_{s, t}$ is irreducible.

Proof. Let $\chi_{s, t} \in \widehat{\mathcal{B}}^{+}, k \in \mathbb{Z}$. Since $C_{q}$ is in the centralizer of $\mathcal{A}$, we have

$$
\chi_{s, t}^{k}\left(C_{q}\right)=\frac{\chi_{s, t}\left(E^{k^{*}} C_{q} E^{k}\right)}{\chi_{s, t}\left(E^{k^{*}} E^{k}\right)}=\frac{\chi_{s, t}\left(E^{k^{*}} E^{k}\right) \chi_{s, t}\left(C_{q}\right)}{\chi_{s, t}\left(E^{k^{*}} E^{k}\right)}=\chi_{s, t}\left(C_{q}\right)=s .
$$

The evaluation of $K$ is

$$
\chi_{s, t}^{k}(K)=\frac{\chi_{s, t}\left(E^{k^{*}} K E^{k}\right)}{\chi_{s, t}\left(E^{k^{*}} E^{k}\right)}=\frac{\chi_{s, t}\left(E^{k^{*}} E^{k}\right) q^{k} \chi_{s, t}(K)}{\chi_{s, t}\left(E^{k^{*}} E^{k}\right)}=q^{k} \chi_{s, t}(K)=q^{k} t .
$$

Suppose $\chi_{s^{\prime}, t^{\prime}} \in \widehat{\mathcal{B}}^{+}$and $\chi_{s^{\prime}, t^{\prime}}=\chi_{s, t}^{k}$. Then, by the above calculation, we get $t^{\prime}=q^{k} t$. Therefore, we have $\chi_{s^{\prime}, t^{\prime}} \neq \chi_{s, t}^{k}$ if $k \neq 0$. This shows that the stabilizer is trival. 
1.3. Classical $*$-Representations of $\mathcal{U}_{q}\left(\mathbf{s u}_{1,1}\right)$. For every $\varepsilon \in[0,1)$ define $\mathbb{Z}_{\varepsilon}:=\{\nu \in \mathbb{R} \mid \nu=z+\varepsilon, z \in \mathbb{Z}\}$. The classical $*$-representations are denoted by $T_{a \varepsilon}$ with indices $a \in \mathbb{R}, \varepsilon \in[0,1)$.

For each $*$-representation $T_{a \varepsilon}$ there is a corresponding set $Z_{a \varepsilon} \subset \mathbb{Z}_{\varepsilon}$ and orthonormal vectors $\left\{b_{\nu}\right\}_{\nu \in Z_{a \varepsilon}}$ such that the domain of $T_{a \varepsilon}$ is $\mathcal{D}\left(T_{a \varepsilon}\right):=\operatorname{span}\left\{b_{\nu}\right\}_{\nu \in Z_{a \varepsilon}}$.

The $*$-representation $T_{a \varepsilon}$ acts on $b_{\nu} \in \mathcal{D}\left(T_{a \varepsilon}\right)$ by

$$
\begin{aligned}
T_{a \varepsilon}(K) b_{\nu} & =q^{\nu} b_{\nu}, \\
T_{a \varepsilon}(E) b_{\nu} & =([a+\nu+1][-a+\nu])^{\frac{1}{2}} b_{\nu+1} \\
& =\left(\frac{q^{2 \nu+1}+\frac{1}{q^{2 \nu+1}}-\left(q^{2 a+1}+\frac{1}{q^{2 a+1}}\right)}{\left(q-q^{-1}\right)^{2}}\right)^{\frac{1}{2}} b_{\nu+1}, \\
T_{a \varepsilon}(F) b_{\nu} & =-([-a+\nu-1][a+\nu])^{\frac{1}{2}} b_{\nu-1}
\end{aligned}
$$

where $b_{\nu}=0$ if $\nu \notin Z_{a \varepsilon}$.

One sees directly that for each $*$-representation $T_{a \varepsilon}$ there is a $*$-representation $\tilde{T}_{a \varepsilon}$ with the only difference to $T_{a \varepsilon}$ being

$$
\tilde{T}_{a \varepsilon}(K) b_{\nu}=-q^{\nu} b_{\nu} .
$$

We call $T_{a \varepsilon}$ a $*$-representation of type one (type two) if the operator $T_{a \varepsilon}(K)$ has positive (negative) eigenvalues. In [2] the $*$-representations of type two were left out.

The duality between *-representations of type one and type two also shows up in the induced $*$-representations described in the previous subsection. For a character $\chi_{s, t}$, the positivity does not depend on the leading sign of $t$ (see (1.3) and (1.4)). Hence, for each induced $*$-representation $\pi_{s, t}$ there is an induced *-representation $\pi_{s,-t}$.

Obviously, if a classical $*$-representation of type one is unitarily equivalent to an induced $*$-representation $\pi_{s, t}$ with $t \in \mathbb{R}^{+}$, the corresponding $*$-representation of type two is unitarily equivalent to $\pi_{s,-t}$.

We restrict our analysis to $*$-representations of type one and induced $*$-representations with positive parameter $t$.

In [2] Burban and Klymik computed the following series of equivalence classes of irreducible $*$-representations, which are all well-behaved irreducible $*$-representations of type one up to unitary equivalence:

We denote both the equivalence class and the representative by $T_{a \varepsilon}$.

- The principal unitary series $T_{a \varepsilon}$ acts on $\left\{b_{\nu}\right\}_{\nu \in \mathbb{Z}_{\varepsilon}}$ with

$$
a=i \frac{\rho}{h}-\frac{1}{2} \text { for } \rho \in[0, \pi], 0 \leq \varepsilon<1, \varepsilon \neq \frac{1}{2}
$$

or

$$
a=i \frac{\rho}{h}-\frac{1}{2} \quad \text { for } \rho \in(0, \pi], \varepsilon=\frac{1}{2}
$$

where we identify $q=e^{\frac{h}{2}}$. 
- The strange series $T_{a \varepsilon}$ acts on $\left\{b_{\nu}\right\}_{\nu \in \mathbb{Z}_{\varepsilon}}$ with

$$
\begin{aligned}
& 0 \leq \varepsilon<1, \\
& \Im a=\frac{\pi}{h}, \quad \Re a>-\frac{1}{2}
\end{aligned}
$$

where we identify $q=e^{\frac{h}{2}}$.

- The supplementary series $T_{a \varepsilon}$ acts on $\left\{b_{\nu}\right\}_{\nu \in \mathbb{Z}_{\varepsilon}}$ with

$$
\begin{aligned}
0 & \leq \varepsilon<\frac{1}{2} & \text { and } & \varepsilon-1<a<-\frac{1}{2} \\
\text { or } & \frac{1}{2}<\varepsilon<1 & \text { and } & -\frac{1}{2}<a<\varepsilon-1 .
\end{aligned}
$$

- The discrete series are

$$
\begin{aligned}
& T_{a}^{+}:=T_{a \varepsilon} \text { with } a>-1, \varepsilon=a \bmod \mathbb{Z}, \varepsilon \in[0,1), \\
& \text { acting on }\left\{b_{\nu}\right\}_{\nu>a}
\end{aligned}
$$

and $\quad T_{a}^{-}:=T_{-a, \varepsilon}$ with $a<1, \varepsilon=a \bmod \mathbb{Z}, \varepsilon \in[0,1)$, acting on $\left\{b_{\nu}\right\}_{\nu<a}$.

Remark 1.7. In the literature one can find slightly different parameters for the discrete and the supplementary series.

1.4. Inducing Procedure - Second Part. Since we restricted ourselves to characters $\chi_{s, t}$ with $t>0$, we can write the parameter $t$ uniquely as $t=q^{\varepsilon+l}$ with $\varepsilon \in[0,1), l \in \mathbb{Z}$, and parametrize the characters by the triplet $(s, \varepsilon, l)$. Therefore, each positive character $\chi$ with $\chi(K)>0$ can be denoted by $\chi_{s, t}$ or $\chi_{s, \varepsilon, l}$. In the following, we will use this duality freely. Hence, for every introduced character $\chi_{s, t}$, we assume the existence of a tuple $(\varepsilon, l) \in[0,1) \times \mathbb{Z}$ which fulfills $t=q^{\varepsilon+l}$.

The condition for the positivity of a character $\chi_{s, t}$ can be written in a more compact form by combining (1.3) and (1.4) to

$$
\chi_{s, t}\left(E^{* z} E^{z}\right)=\prod_{k=1}^{|z|}\left(\frac{q^{2 k-1} t^{2 \operatorname{sgn}(z)}+q^{-2 k+1} t^{-2 \operatorname{sgn}(z)}}{\left(q-q^{-1}\right)^{2}}-s\right) \geq 0
$$

for all $z \in \mathbb{Z}$.

Therefore, a sufficient and, if all factors of (1.17) are nonzero, necessary condition on $s$ for $\chi_{s, t}$ being positive is

$$
s<\min _{z \in \mathbb{Z}} \frac{q^{2 z-1} t^{2}+\frac{1}{q^{2 z-1} t^{2}}}{\left(q-q^{-1}\right)^{2}} .
$$

Due to the duality introduced above, we can state the following lemma.

Lemma 1.8. Let $\chi_{s, t} \in \widehat{\mathcal{B}}^{+}$. Then we have

$$
\min _{z \in \mathbb{Z}} \frac{q^{2 z-1} t^{2}+\frac{1}{q^{2 z-1} t^{2}}}{\left(q-q^{-1}\right)^{2}}=\frac{q^{2 \varepsilon-1}+\frac{1}{q^{2 \varepsilon-1}}}{\left(q-q^{-1}\right)^{2}} \geq \frac{2}{\left(q-q^{-1}\right)^{2}} .
$$


Proof. A short analysis of the function $f: \mathbb{R}_{\neq 0}^{+} \rightarrow \mathbb{R}$ given by $f(x):=x+\frac{1}{x}$ shows a global minimum at $x=1$.

With this, the minimum of the nominator of the left hand term of (1.19) is

$$
\min _{z \in \mathbb{Z}} q^{2 z-1} t^{2}+\frac{1}{q^{2 z-1} t^{2}}=\min _{z \in \mathbb{Z}} q^{2 z+2 l+2 \varepsilon-1}+\frac{1}{q^{2 z+2 l+2 \varepsilon-1}}=q^{2 \varepsilon-1}+\frac{1}{q^{2 \varepsilon-1}} .
$$

From $(1.17),(1.18)$ and Lemma 1.8, we can deduce the following two corollaries.

Corollary 1.9. Let $\chi_{s, t} \in \widehat{\mathcal{B}}^{+}$. The parameter $s$ fulfills either

$$
s=\frac{q^{2 z-1} t^{2}+\frac{1}{q^{2 z-1} t^{2}}}{\left(q-q^{-1}\right)^{2}} \text { for } z \in \mathbb{Z} \quad \text { or } \quad s<\frac{q^{2 \varepsilon-1}+\frac{1}{q^{2 \varepsilon-1}}}{\left(q-q^{-1}\right)^{2}} .
$$

Corollary 1.10. Let $\chi_{s, t} \in \widehat{\mathcal{B}}^{+}$. If $s$ fulfills the inequality

$$
s<\frac{q^{2 \varepsilon-1}+\frac{1}{q^{2 \varepsilon-1}}}{\left(q-q^{-1}\right)^{2}}
$$

we have $\mathcal{D}_{\chi_{s, t}}=\mathbb{Z}$.

First, we discuss the second case of Corollary 1.9.

Lemma 1.11. The unitary equivalence classes of all induced *-representations by positive characters $\chi_{s, t}$ with $s<\frac{q^{2 \varepsilon-1}+\frac{1}{q^{2 \varepsilon-1}}}{\left(q-q^{-1}\right)^{2}}$ can be parametrized by the set $[0,1) \times(-\infty, 0)$.

A parametrization is given by $(\zeta, x) \mapsto\left[\pi_{s, t}\right]$ with

$$
\begin{aligned}
t & =q^{\zeta}, \\
\text { and } s & =x+\frac{q^{2 \zeta-1}+\frac{1}{q^{2 \zeta-1}}}{\left(q-q^{-1}\right)^{2}} .
\end{aligned}
$$

Each such character $\chi_{s, t}$ has the orbit $\operatorname{Orb} \chi_{s, t}=\left\{\chi_{s, q^{k} t} \mid k \in \mathbb{Z}\right\}$, and the index set $M_{s, t}$ of the orthonormal basis $\left\{e_{k}\right\}_{k \in M_{s, t}}$ (see (1.5)) is $\mathbb{Z}$.

Proof. If $\chi_{s, t}$ is in $\mathcal{D}_{k}$, we have $\chi_{s, t}^{k}=\chi_{s, q^{k} t}$. Therefore, two characters $\chi_{s, t}, \chi_{s^{\prime}, t}$ are in different orbits for $s \neq s^{\prime}$.

Recall that for a character $\chi_{s, t}$ with $t=q^{\varepsilon+l}$ the minimum in (1.19) only depends on $\varepsilon$. Taking into account the partial action, we see that $\chi_{s, t}$ is in the same orbit as $\chi_{s, q^{k} t}$ for all $k \in \mathbb{Z}$ if $s<\frac{q^{2 \varepsilon-1}+\frac{1}{q^{2 \varepsilon-1}}}{\left(q-q^{-1}\right)^{2}}$.

Combining both arguments for the parameters shows that the stated mapping in the assertion is a parametrization and that $\operatorname{Orb}_{\chi_{s, t}}=\left\{\chi_{s, q^{k} t} \mid k \in \mathbb{Z}\right\}$.

Since $\chi_{s, t}\left(E^{* z} E^{z}\right)$ in (1.17) is equal to $\left\|E^{z} \otimes 1\right\|^{2}$ for all $z \in \mathbb{Z}$, the index set $M_{s, t}$ is $\mathbb{Z}$ for $s$ fulfilling (1.18).

Proposition 1.12. The equivalence classes $\left[\pi_{s, q^{\varepsilon}}\right]$ with the parameters

$$
\varepsilon \in[0,1) \backslash\left\{\frac{1}{2}\right\} \text { and } \frac{-2}{\left(q-q^{-1}\right)^{2}} \leq s \leq \frac{2}{\left(q-q^{-1}\right)^{2}}
$$


or

$$
\varepsilon=\frac{1}{2} \text { and } \frac{-2}{\left(q-q^{-1}\right)^{2}} \leq s<\frac{2}{\left(q-q^{-1}\right)^{2}}
$$

are unitarily equivalent to the principal unitary series.

Proof. Combining (1.19) with Lemma 1.11, we see that the equivalence classes stated in the assertion are a subset of the equivalence classes parametrized in Lemma 1.11.

Let $T_{a \varepsilon}$ be an equivalence class of the principal unitary series. We set

$$
t:=q^{\varepsilon}
$$

and

$$
s:=\frac{q^{2 a+1}+\frac{1}{q^{2 a+1}}}{\left(q-q^{-1}\right)^{2}}=\frac{e^{i \rho}+e^{-i \rho}}{\left(q-q^{-1}\right)^{2}}=\frac{2 \Re e^{i \rho}}{\left(q-q^{-1}\right)^{2}} .
$$

This implies for $\varepsilon \neq \frac{1}{2}$ by (1.9) that $\frac{-2}{\left(q-q^{-1}\right)^{2}} \leq s \leq \frac{2}{\left(q-q^{-1}\right)^{2}}$ and for $\varepsilon=\frac{1}{2}$ by (1.10) that $\frac{-2}{\left(q-q^{-1}\right)^{2}} \leq s<\frac{2}{\left(q-q^{-1}\right)^{2}}$. Therefore $\chi_{s, t}$ is a positive character and the induced $*$-representation $\pi_{s, t}$ exists.

We define a unitary operator $U: \mathcal{H}\left(\pi_{s, t}\right) \rightarrow \mathcal{H}\left(T_{a \varepsilon}\right)$ by setting

$$
U\left(e_{j}\right)=b_{j+\varepsilon}
$$

for all $j \in \mathbb{Z}$.

This operator is well-defined since $\mathcal{H}\left(\pi_{s, t}\right)=\overline{\operatorname{span}}\left\{e_{k}\right\}_{k \in \mathbb{Z}}$ and for an operator of the principal unitary series we have $\mathcal{H}\left(T_{a \varepsilon}\right)=\overline{\operatorname{span}}\left\{b_{\nu}\right\}_{\nu \in \mathbb{Z}_{\varepsilon}}$.

Comparing the action of $T_{a \varepsilon}$ in (1.6)-(1.8) with the action of $\pi_{s, t}$ in Proposition 1.5 , we see that $U^{-1} T_{a \varepsilon} U=\pi_{s, t}$ if and only if

$$
\begin{aligned}
& t=q^{\varepsilon}, \\
& s=\frac{q^{2 a+1}+\frac{1}{q^{2 a+1}}}{\left(q-q^{-1}\right)^{2}} .
\end{aligned}
$$

Since we defined $s$ and $t$ this way, $T_{a \varepsilon}$ and $\pi_{s, t}$ are unitarily equivalent.

We see that the construction above maps the principal unitary series bijectively on the stated equivalence classes in the assertion if we vary the parameters of the principal unitary series. This shows the assertion.

Proposition 1.13. The equivalence classes $\left[\pi_{s, q^{\varepsilon}}\right]$ with the parameters

$$
\varepsilon \in[0,1) \text { and } s<\frac{-2}{\left(q-q^{-1}\right)^{2}}
$$

are unitarily equivalent to the strange series.

Proof. The proof is the same as the proof of the previous Proposition with the only difference being that for an operator $T_{a \varepsilon}$ of the strange series we get by 
(1.12):

$$
\begin{aligned}
s= & \frac{q^{2 a+1}+\frac{1}{q^{2 a+1}}}{\left(q-q^{-1}\right)^{2}}=\frac{q^{2 i \frac{\pi}{h}} q^{2 \Re a+1}+\frac{1}{q^{2 i \frac{\pi}{h}} q^{2 \Re a+1}}}{\left(q-q^{-1}\right)^{2}}= \\
& =\frac{e^{i \pi} q^{2 \Re a+1}+\frac{1}{e^{i \pi} q^{2 \Re a+1}}}{\left(q-q^{-1}\right)^{2}}=-\frac{q^{2 \Re a+1}+\frac{1}{q^{2 \Re a+1}}}{\left(q-q^{-1}\right)^{2}}<\frac{-2}{\left(q-q^{-1}\right)^{2}} .
\end{aligned}
$$

Proposition 1.14. The equivalence classes $\left[\pi_{s, q^{\varepsilon}}\right]$ with the parameters

$$
\varepsilon \in[0,1) \backslash\left\{\frac{1}{2}\right\} \text { and } \frac{2}{\left(q-q^{-1}\right)^{2}}<s<\frac{q^{2 \varepsilon-1}+\frac{1}{q^{2 \varepsilon-1}}}{\left(q-q^{-1}\right)^{2}}
$$

are unitarily equivalent to the supplementary series.

Proof. Let $T_{a \varepsilon}$ be an equivalence class of the supplementary series. We set

$$
t:=q^{\varepsilon}, \quad s:=\frac{q^{2 a+1}+\frac{1}{q^{2 a+1}}}{\left(q-q^{-1}\right)^{2}} .
$$

The parameters of the supplementary series fulfill

$$
\begin{gathered}
\varepsilon-1<a<-\frac{1}{2} \text { and } 0 \leq \varepsilon<\frac{1}{2}, \\
\text { or } \quad-\frac{1}{2}<a<\varepsilon-1 \text { and } \frac{1}{2}<\varepsilon<1 .
\end{gathered}
$$

For the rest of the proof keep in mind the proof of Lemma 1.8.

If (1.24) holds, we have

$$
|2 \varepsilon-1|=1-2 \varepsilon>|2 a+1|=-2 a-1 \text { since } a>\varepsilon-1 .
$$

If (1.25) holds, we have

$$
|2 \varepsilon-1|=2 \varepsilon-1>|2 a+1|=2 a+1 \text { since } a<\varepsilon-1 .
$$

Therefore, $s$ fulfills inequality (1.18) and $\chi_{s, t}$ is a positive character. We introduce the unitary operator $U$ as defined in (1.20), which shows the unitary equivalence of $\pi_{s, t}$ and $T_{a \varepsilon}$.

Again, as in the previous two proofs, we vary the parameters of the supplementary series and show the surjectivity of the above construction of $\pi_{s, t}$ :

If $0 \leq \varepsilon<\frac{1}{2}$, we have $2 \varepsilon-1<0$. Therefore, varying $a$ gives all parameters $s$ fulfilling (1.23) since $2 \varepsilon-1<2 a+1<0$ is equivalent to $\varepsilon-1<a<-\frac{1}{2}$. If $\frac{1}{2}<\varepsilon<1$, we can argue in an analog way with inverted inequalities.

Remark 1.15. With the last three propositions we have assigned all induced *-representations from the second case of Corollary 1.9.

To find induced $*$-representations fitting to the discrete series, we have to consider the induced $*$-representations $\pi_{s, t}\left(\right.$ recall $\left.t=q^{l+\varepsilon}, \varepsilon \in[0,1), l \in \mathbb{Z}\right)$ with

$$
s=\frac{q^{2 z-1} t^{2}+\frac{1}{q^{2 z-1} t^{2}}}{\left(q-q^{-1}\right)^{2}}=\frac{q^{2(l+z)+2 \varepsilon-1}+\frac{1}{q^{2(l+z)+2 \varepsilon-1}}}{\left(q-q^{-1}\right)^{2}}
$$


for $z \in \mathbb{Z}$, which have not been parametrized or assigned to classical $*$-representations yet.

Lemma 1.16. Let $\varepsilon \in[0,1)$. If $l \in \mathbb{Z}_{\leq-1}$, equation (1.26) is fulfilled for an induced $*$-representation $\pi_{s, t}$

$$
\begin{aligned}
\text { for } \varepsilon & \in(0,1) & \text { only if } 0<z \leq-l \\
\text { and for } \varepsilon & =0 & \text { only if } 0<z \leq-l+1 .
\end{aligned}
$$

If $l \in \mathbb{Z}_{\geq 0}$, equation (1.26) is fulfilled for an induced $*$-representation $\pi_{s, t}$

$$
\begin{aligned}
\text { for } \varepsilon & \in(0,1) & & \text { only if }-l \leq z \leq 0 \\
\text { and for } \varepsilon & =0 & & \text { only if }-l \leq z \leq 0 \text { or }-l \leq z \leq-l+1 .
\end{aligned}
$$

Proof. The assertion is shown by straight forward computation.

Proposition 1.17. Let $t \in \mathbb{R}_{>0} \backslash\{1\}$. The unitary equivalence classes of all induced $*$-representations $\pi_{s, t}$ with $s$ fulfilling

$$
s=\frac{q^{2 z-1} t^{2}+\frac{1}{q^{2 z-1} t^{2}}}{\left(q-q^{-1}\right)^{2}}, z \in \mathbb{Z},
$$

can be parametrized by $[0,1) \times \mathbb{Z} \backslash\{(0,0)\}$. A parametrization is given by $(\zeta, r) \mapsto$ $\left[\pi_{s, t}\right]$ with

$$
\begin{aligned}
& t=q^{r+\zeta}, \\
& s=\frac{q^{2(r+\zeta)-\operatorname{sgn}\left(r+\frac{1}{2}\right)}+\frac{1}{q^{2(r+\zeta)-\operatorname{sgn}\left(r+\frac{1}{2}\right)}}}{\left(q-q^{-1}\right)^{2}}
\end{aligned}
$$

acting as described in Proposition 1.5 on the orthonormal basis $\left\{e_{k}\right\}_{k<1}$ for $r \leq-1$ and on $\left\{e_{k}\right\}_{k>-1}$ for $r \geq 0$.

If $t=1$, there is only the induced *-representation $\pi_{s, 1}$ with $s=\frac{q+\frac{1}{q}}{\left(q-q^{-1}\right)^{2}}$ fulfilling (1.27). It acts on $\mathcal{H}\left(\pi_{s, 1}\right)=\operatorname{span}\left\{e_{0}\right\}$ by the operators $\pi_{s, 1}(K)=\mathbf{1}$ and $\pi_{s, 1}(E)=\pi_{s, 1}(F)=0$.

Proof. Let $\varepsilon \in[0,1)$ and $l \in \mathbb{Z}_{\leq-1}$. Define $t:=q^{\varepsilon+l}$.

Suppose

$$
s=\frac{q^{2(z+l)+2 \varepsilon-1}+\frac{1}{q^{2(z+l)+2 \varepsilon-1}}}{\left(q-q^{-1}\right)^{2}}
$$

where $z \in \mathbb{N}$ with $0<z \leq-l$ for $\varepsilon \in(0,1)$ and $0<z \leq-l+1$ for $\varepsilon=0$.

By Lemma 1.16 all positive characters $\chi_{s, t}$ fulfilling (1.26) have a parameter $z$ which is of this form. Moreover, since for $\varepsilon=0$ the parameter $s$ is the same for $z=-l+1$ and $z=-l$, we can assume $0<z \leq-l$ in this case too. 
Now, for the character $\chi_{s, t}$ and $n \geq z$, we have

$$
\begin{aligned}
\chi_{s, t}\left(E^{* n} E^{n}\right) & =\prod_{k=1}^{n}\left(\frac{q^{2 k-1} t^{2}+q^{-2 k+1} t^{-2}}{\left(q-q^{-1}\right)^{2}}-s\right) \\
& =\prod_{k=1}^{n}\left(\frac{q^{2(k+l)+2 \varepsilon-1}+\frac{1}{q^{2(k+l)+2 \varepsilon-1}}}{\left(q-q^{-1}\right)^{2}}-s\right)=0
\end{aligned}
$$

and $s$ fulfills

$$
s<\min _{k<z} \frac{q^{2 k-1} t^{2}+\frac{1}{q^{2 k-1} t^{2}}}{\left(q-q^{-1}\right)^{2}} .
$$

This shows that $\chi_{s, t}$ is a positive character. Further, we have $E^{k} \otimes 1=0 \Longleftrightarrow k \geq$ $z$, and $\left\{e_{k}\right\}_{k<z}, e_{k}:=\frac{E^{k} \otimes 1}{\left\|E^{k} \otimes 1\right\|}$, is the orthonormal basis of $\mathcal{H}\left(\pi_{s, t}\right)$ (see Proposition $1.5)$.

Therefore, the partial action $\chi_{s, t}^{k}=\chi_{s, q^{k} t}$ is defined for all $k<z$, and the induced $*$-representation $\pi_{s, q^{k} t}$ acts on $e_{n}$ with $n<z-k \in[1, \infty)$. Setting $k=z-1$, we see that

$$
\pi_{s, t} \in\left[\pi_{s, q^{l+z-1+\varepsilon}}\right]
$$

where $\pi_{s, q^{l+z-1+\varepsilon}}$ acts on $e_{n}$ with $n<1$.

Combining (1.30) with the partial action from Proposition 1.6, we can conclude that two characters $\chi_{s_{1}, t_{1}}$ and $\chi_{s_{2}, t_{2}}$ with parameters $\varepsilon_{1}=\varepsilon_{2} \in[0,1), l_{1}, l_{2} \in \mathbb{Z}_{\leq-1}$,

$$
s_{1}=\frac{q^{2\left(z_{1}+l_{1}\right)+2 \varepsilon_{1}-1}+\frac{1}{q^{2\left(z_{1}+l_{1}\right)+2 \varepsilon_{1}-1}}}{\left(q-q^{-1}\right)^{2}}, 0<z_{1} \leq-l_{1},
$$

and

$$
s_{2}=\frac{q^{2\left(z_{2}+l_{2}\right)+2 \varepsilon_{2}-1}+\frac{1}{q^{2\left(z_{2}+l_{2}\right)+2 \varepsilon_{2}-1}}}{\left(q-q^{-1}\right)^{2}}, 0<z_{2} \leq-l_{2},
$$

are in the same orbit if and only if $s_{1}=s_{2}$. This implies $z_{1}+l_{1}=z_{2}+l_{2}$ for $\varepsilon_{1} \neq 0$ and, since $z_{1}+l_{1} \leq 0, z_{2}+l_{2} \leq 0$, it implies $z_{1}+l_{1}=z_{2}+l_{2}$ for $\varepsilon_{1}=0$ too. Hence, the induced $*$-representations $\pi_{s_{1}, t_{1}}$ and $\pi_{s_{2}, t_{2}}$ are unitarily equivalent if and only if $z_{1}+l_{1}=z_{2}+l_{2}$.

Therefore, the equivalence classes for $l \leq-1$ and fixed $\varepsilon$ can be parametrized by $z+l-1$. That is, we can define a parametrization $(\zeta, r) \mapsto\left[\pi_{s, t}\right]$ on $[0,1) \times \mathbb{Z}_{\leq-1}$ given by

$$
t=q^{r+\zeta}, \quad s=\frac{q^{2(r+\zeta)+1}+\frac{1}{q^{2(r+\zeta)+1}}}{\left(q-q^{-1}\right)^{2}},
$$

where $\pi_{s, t}$ acts on $\left\{e_{k}\right\}_{k<1}$.

A similar argumentation shows the case $\varepsilon \in[0,1)$ and $l \in \mathbb{Z}_{\geq 0}$.

Proposition 1.18. The unitary equivalence classes parametrized in Proposition 1.17 are unitarily equivalent to the discrete series. 
By terms of that parametrization, the discrete series $T_{a}^{-}$are unitarily equivalent to the induced unitary equivalence classes with parameters $(\zeta, r) \in[0,1) \times \mathbb{Z}_{\leq-1}$, and the discrete series $T_{a}^{+}$are unitarily equivalent to the induced unitary equivalence classes with parameters $(\zeta, r) \in[0,1) \times \mathbb{Z}_{\geq 0} \backslash\{(0,0)\}$.

Proof. We use the parametrization of Proposition 1.17. Let $(\zeta, r)$ be in $[0,1) \times$ $\mathbb{Z}_{\leq-1}$ and suppose $\left[\pi_{s, t}\right]$ is the corresponding induced equivalence class for the parameters $(\zeta, r)$.

Choose $z_{0}, k_{0} \in \mathbb{Z}$ such that $z_{0}-k_{0}=r+1$. Let $\left\{b_{\nu}\right\}_{\nu \in I_{\zeta, r}}$ be an orthonormal basis with an index set $I_{\zeta, r} \subset \mathbb{Z}_{\zeta}$ defined by

$$
I_{\zeta, r}:=\left\{z_{0}-1+\zeta+j \mid j \in \mathbb{Z}, z_{0}-1+\zeta+j<r+1+\zeta\right\} .
$$

For all $j \in \mathbb{Z}$ the inequality $z_{0}-1+\zeta+j<r+1+\zeta$ is equivalent to $k_{0}+j<1$.

Next, we define a unitary operator $U: \mathcal{D}\left(\pi_{s, t}\right) \rightarrow \operatorname{span}\left\{b_{\nu}\right\}_{\nu \in I_{\zeta, r}}$ given by

$$
e_{k_{0}+j} \mapsto b_{z_{0}-1+\zeta+j}
$$

for all $j \in \mathbb{Z}$ with $k_{0}+j<1$.

The *-representation $U \pi_{s, t} U^{-1}$ is $T_{a}^{-}$with $a=r+\zeta+1$ and $\varepsilon=\zeta$ : $U \pi_{s, t}(K) U^{-1}$ acts on $b_{\nu}$ by

$$
\begin{aligned}
& U \pi_{s, t}(K) U^{-1} b_{z_{0}-1+\zeta+j}=U \pi_{s, t}(K) e_{k_{0}+j}= \\
& U q^{k_{0}+j} q^{r+\zeta} e_{k_{0}+j}=q^{k_{0}+j} q^{r+\zeta} b_{z_{0}-1+\zeta+j}=q^{z_{0}-1+\zeta+j} b_{z_{0}-1+\zeta+j}
\end{aligned}
$$

$U \pi_{s, t}(E) U^{-1}$ acts on $b_{\nu}$ by

$$
\begin{aligned}
& U \pi_{s, t}(E) U^{-1} b_{z_{0}-1+\zeta+j}=U \pi_{s, t}(E) e_{k_{0}+j}= \\
& U\left(\frac{q^{2\left(k_{0}+j\right)+1}\left(q^{r+\zeta}\right)^{2}+q^{-2\left(k_{0}+j\right)-1}\left(q^{r+\zeta}\right)^{-2}}{\left(q-q^{-1}\right)^{2}}-\frac{q^{2(r+\zeta)+1}+\frac{1}{q^{2(r+\zeta)+1}}}{\left(q-q^{-1}\right)^{2}}\right)^{\frac{1}{2}} e_{k_{0}+j+1}= \\
& \left(\frac{q^{2\left(k_{0}+j\right)+1}\left(q^{r+\zeta}\right)^{2}+q^{-2\left(k_{0}+j\right)-1}\left(q^{r+\zeta}\right)^{-2}}{\left(q-q^{-1}\right)^{2}}-\frac{q^{2(r+\zeta)+1}+\frac{1}{q^{2(r+\zeta)+1}}}{\left(q-q^{-1}\right)^{2}}\right)^{\frac{1}{2}} b_{z_{0}-1+\zeta+j+1} .
\end{aligned}
$$

In the left fraction of the term above, we can replace $k_{0}+j+r+\zeta$ by $z_{0}-1+\zeta+j$. In the right fraction the sum $r+\zeta$ can be replaced by $-r-\zeta-1$. Hence, we see the equality between $U \pi_{s, t} U^{-1}$ and $T_{-r-\zeta-1, \zeta}$ acting on $\left\{b_{\nu} \mid \nu<r+\zeta+1\right\}$, which is $T_{r+\zeta+1}^{-}$.

The map $[0,1) \times \mathbb{Z}_{\leq-1} \rightarrow(-\infty, 1)$ with $(\zeta, r) \mapsto r+\zeta+1$ is a bijection. Therefore, the discrete series $T_{a}^{-}$are unitarily equivalent to the unitary equivalence classes parametrized by $\zeta \in[0,1), r \in \mathbb{Z}_{\leq-1}$.

A similar argumentation shows the assertion for $(\zeta, r) \in[0,1) \times \mathbb{Z}_{\geq 0} \backslash\{(0,0)\}$.

Summing up, we get the following theorem. Recall the duality introduced in subsection 1.3.

Theorem 1.19. Let $\left\{\mathcal{A}_{z}\right\}_{z \in \mathbb{Z}}$ be the $\mathbb{Z}$-grading on $\mathcal{U}_{q}\left(\right.$ su $\left.u_{1,1}\right)$ defined by $E \in \mathcal{A}_{1}$, $K, K^{-1} \in \mathcal{A}_{0}$. 
Then there is a one-to-one correspondence between the induced equivalence classes of *-representations by characters and the classical (or well-behaved) equivalence classes of $*$-representations.

Proposition 1.12, Proposition 1.13, Proposition 1.14 and Proposition 1.18 assign subfamilies of the induced equivalence classes with positive second parameter to the corresponding classical series of well-behaved $*$-representations of type one.

\section{REFERENCES}

1. J.-P. Antoine, A. Inoue and C. Trapani, Partial $*$-Algebras and their Operator Realizations, Mathematics and its Applications 553, Kluwer Academic Publishers, Dordrecht, 2002.

2. I.M. Burban and A.U. Klimyk, Representation of the Quantum Algebra $\mathcal{U}_{q}\left(s u_{1,1}\right)$, J. Phys. A: Math. Gen. 26 (1993), 2139-2151.

3. P.A. Dowerk and Y. Savchuk, Induced $*$-Representations and $C^{*}$-Envelopes of Some Quantum *-Algebras., J. Lie Theory 23 (2013), No. 1, 229-250.

4. J.M.G. Fell and R.S. Doran, Representations of *-Algebras, Locally Compact Groups, and Banach *-Algebraic Bundles, Academic Press, Inc., Boston, MA, 1988.

5. G. Frobenius, Über Relationen zwischen den Charakteren einer Gruppe und denen ihrer Untergruppen, Sitz. Preuss. Akad. Wiss. (1898), 501-515.

6. A.U. Klimyk and K. Schmüdgen, Quantum Groups and Their Representations, Springer, 1997.

7. G.W. Mackey, Imprimitivity for Representations of Locally Compact Groups I., Proc. Nat. Acad. Sci. U.S.A. 35 (1949), 537-545.

8. V. Ostrovskyi and Yu. Samoilenko, Introduction to the theory of representations of finitely presented *-algebras. I. Representations by bounded operators., Reviews in Mathematics and Mathematical Physics 11 pt.1, Harwood Academic Publishers, Amsterdam, 1999.

9. Y. Savchuk and K. Schmüdgen, Unbounded Induced Representations of *-Algebras, Algebr. Represent. Theory 16 (2013), no. 2, 309-376.

10. S.L. Woronowicz, Twisted SU(2) Group. An Example of a Non-Commutative Differential Calculus, Publ. RIMS Kyoto Univ. 23 (1987), 117-181.

11. K. Schmüdgen, Unbounded Operator Algebras and Representation Theory, Operator Theory: Advances and Applications 37, Birkhäuser Verlag, Basel, 1990.

1 MaX-Planck-Institut für Mathematik in Den Naturwissenschaften, InselStrasse 22, 04103 LeIPZIG, Germany

E-mail address: david.dubray@web.de 